\title{
DER BAUER IN DER FINNISCHEN LANDSCHAFT
}

\author{
Werner Nigg
}

Ein mutiges Volk nahm Besitz von den riesigen Wald-, Seen- und Sumpflandschaften Finnlands. Mutig im harten Kampf gegen die Naturgewalten schuf es Rodungsoasen für Wiesen, Äcker und wohnliche Häuser bis «an den Rand der Welt». Im neuen, im Verlag Kümmerly \& Frey, Bern, erschienenen Buch «Finnland» schildert Prof. Dr. Werner Nigg eine Fahrt durch Städte, Wälder und über Seen von Süden bis ins nördliche Lappland. Außerdem wünscht dieses Werk allerlei Wissenswertes über Aufbau, Klima, Geschichte, Wirtschaft und Kultur des ganzen Landes zu vermitteln. Der nachfolgende Abschnitt sowie die Farbtafel und die Kartenskizzen sind Proben aus ihm.

Ein moderner Triebwagen der Finnischen Staatsbahnen bringt uns von Rovaniemi nach Kemijärvi in finnisch Lappland. Wir haben im hintersten Abteil, das wie ein halbrundes Pavillon mit großen Fenstern aussieht, gemütliche Plätze gefunden. Leicht und fast geräuschlos rollt unser Zug über die Ounaskoski- und Suutarinkorvabrücke, wo wir die Mündung des breiten, ziemlich reißenden Ounaskoski in den Kemijoki sehen. Der Wald ist relativ licht, und das helle Graugrün der Flechten wird streckenweise zur dominierenden Farbe. Zwischen den Stationen Kulus und Vika überqueren wir den Polarkreis und befinden uns nun in der «kalten Zone»!- «Kalte, Zone»? Dabei scheint die Sonne vom wolkenlosen Himmel, und in unserem Wagen ist es bei offenen Fenstern so warm, daß verschiedene Passagiere hemdärmelig dasitzen!

Weit und breit keine Siedlung. Man könnte glauben, außerhalb der bewohnten Welt zu sein. Doch bald halten wir bei der Endstation, beim stattlichen Ort Kemijärvi, der nach den Zerstörungen durch die deutschen Truppen im Jahre $194+$ nach modernen Gesichtspunkten wieder weitgehend aufgebaut worden ist.

Nun fahren wir mit dem Autobus über eine ziemlich holperige Straße. Rechts und links Wald. Nur in weiten Abständen folgen Rodungen mit neuen Bauernhöfen oder weidenden fahlweißen Kühen.

Dann wechselt das Landschaftsbild. Wir überqueren auf niedrigem Damm einen Waldsee, übersät mit unzähligen weißen und gelben Seerosen, die sich vom grünen Blättergrund abheben. Niedrige Birken spiegeln ihr frischgrünes Laub und ihre blendendweißen Stämme im moorigen Wasser. Im Hintergrund türmen sich bedrohlich graublaue dunkle Wolken, und davor spannen kräftig leuchtende Farben einen mächtigen Regenbogen! - Gäbe es wohl noch eine stimmungsvollere Szenerie für einen Reigen zierlicher Elfen?

Wir halten bei einem Bauernhof an. Der Bäuerin und dem Bauern sieht man die schwere Arbeitslast an, die sie zu tragen haben. Das Gesicht von Sonne, Regen, Wind und Kälte braun gegerbt, ihr Gang kraftvoll und schwerfällig. Mit ungespielter Freundlichkeit zeigen sie uns Haus und Hof.

Sie sind Neusiedler und gehören zu jenen 425000 Menschen, die freiwillig das an die Russen verlorene Gebiet verlassen haben. Hier, etwa $30 \mathrm{~km}$ von der nächsten Ortschaft entfernt, hat ihnen der Staat zu einer neuen Heimstätte verholfen.

Es ist nicht leicht, auf «vorgeschobenem Posten» Landwirt zu sein! Ohne ausreichenden Waldbesitz wäre eine bäuerliche Wirtschaft in dieser Zone überhaupt nicht möglich. Waldarbeit und Holznutzung füllen die Wintermonate aus und bringen etwas Bargeld in den Haushalt. Mühsam werden dem Urwald einige Rodungsflächen abgerungen. Der Ackerbau spielt eine untergeordnete Rolle. Der Schnee liegt sechs bis sieben Monate lang, die frostfreie Zeit dauert zwei bis drei Monate. Dank der hellen Sommernächte braucht die Gerste nur 70 Tage von der Aussaat bis zur Ernte. Sie bildet für die Siedler Lapplands das Hauptgetreide. Daneben werden noch Kartoffeln, Roggen und selten etwas Hafer angepflanzt. Unendlich günstiger sind die Verhältnisse für die Viehzucht. Im Sommer stehen den Tieren Waldweiden zur Verfügung, und die grünen Flußtäler liefern Heu für die lange Stallfütterung im Winter. 

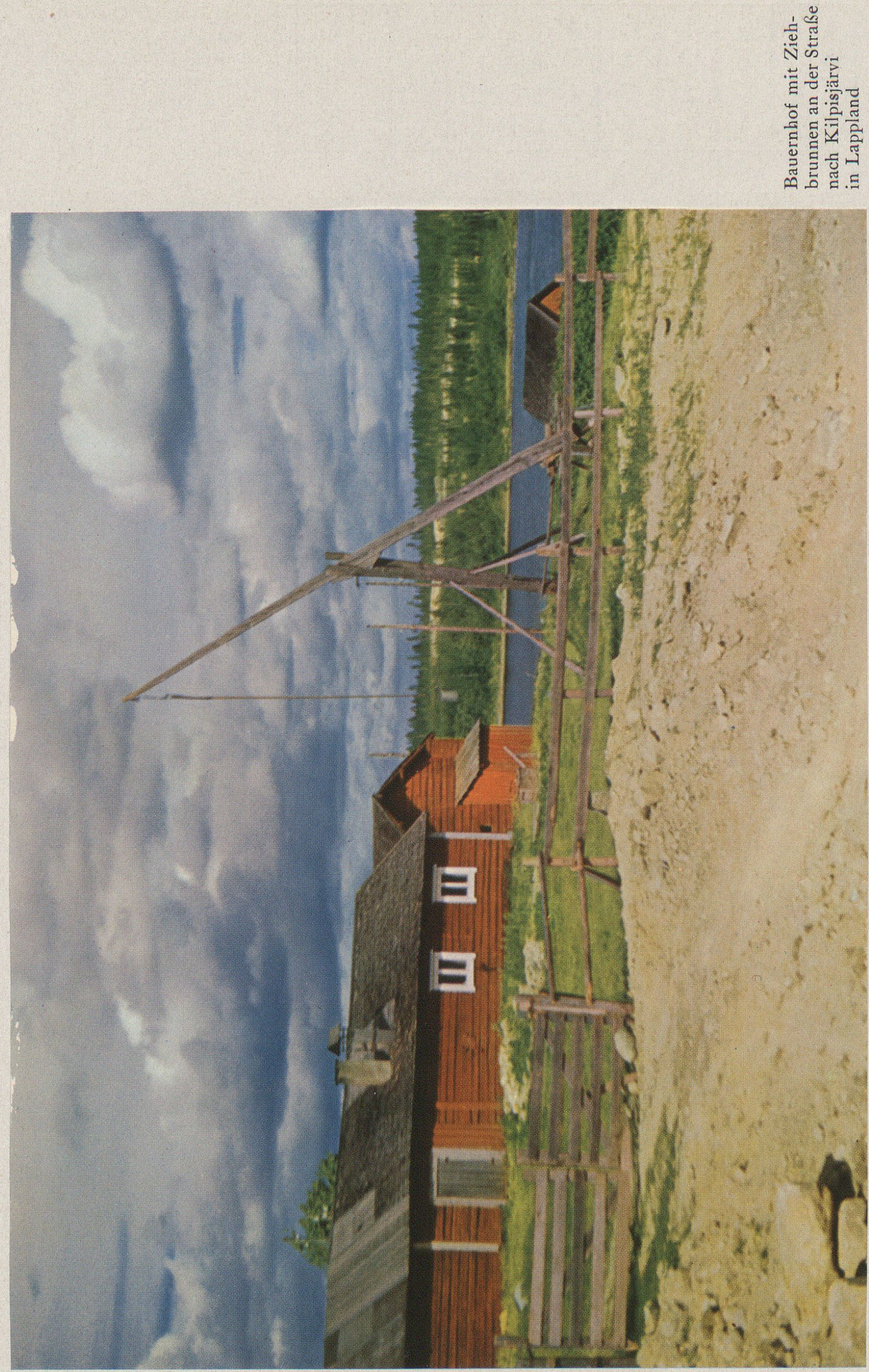


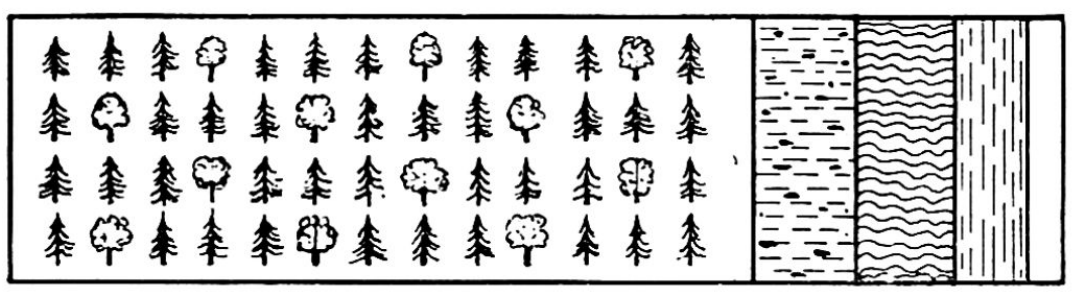

Aufteilung der Gesamtfäche

Wald $71 \%$

Torfmoor $9,8 \%$

Binnengewässer $9,3 \%$

Anbaufläche $7 \%$

übrige $2,9 \%$

(von links nach rechts)

Das Wohnhaus, der Stall mit Tenne, die Vorräte, Gerätehäuser und die Sauna alles Holzbauten - umfassen auf drei Seiten einen Platz, welcher peinlich sauber aufgeräumt ist, wie am Vorabend eines Festtages. Die Anordnung der Gebäude erinnert an den Vierkanthof Skandinaviens.

Das große Wohnhaus mit einem kleinen überdeckten Vorbau macht einen behäbigen Eindruck. Es verfügt über die charakteristischen Merkmale aller finnischen Bauernhäuser: eine Brandleiter zum Dach, vor dem Haus eine große Holzschaukel und ein Ziehbrunnen, dessen langer Arm schräg emporragt.

Die geräumige helle Stube zeugt von gediegener ländlicher Wohnkultur. Ein riesiger gemauerter Ofen nimmt etwa ein Viertel des Raumes ein. Den Tisch schmükken eine handgewobene Leinendecke und ein großer Blumenstrauß. Darüber hängt eine hübsche Petrollampe. Frohe, bunte Vorhänge und zahlreiche Zimmerpflanzen zieren die Fenster. Ein großer selbstgewobener Teppich, ein stattlicher Kasten aus Birkenholz und ein Schaukel-Lehnstuhl bilden das übrige Inventar. Die ganze Ausstattung mit all den vielen Einzelheiten, wie beispielsweise Beschläge an Türen und Kasten, Bilderrahmen, Blumenvasen, und noch vieles andere zeigen den Sinn und guten Geschmack für ein hochstehendes bäuerliches Kunstgewerbe.

Wir können uns gut vorstellen, daß es in der wohnlichen Stube sehr gemütlich sein kann, wenn der große Ofen wohlige Wärme ausstrahlt und man sich, ein Pfeifchen rauchend, im bequemen Schaukelstuhl wiegt, während draußen der Wintersturm heult. Auch die andern Räume sind heimelig. Da steht ein großer Webstuhl, dort ein Spinnrad; beide werden von der Bäuerin noch fleißig benützt.

Stallung und Scheune sind unter einem Dach. Zwei hörnerlose Kühe stehen im schneeweiß gekalkten Stall. Die andern Tiere sind draußen auf der Weide. Durch eine Türe gelangt man in eine Art Küche, die gleichzeitig als Sennerei dient. Auch hier, wie übrigens auf dem ganzen Hofe, herrscht vorbildliche Sauberkeit, wie in einem Musterbetrieb.

41,5\% der Bevölkerung Finnlands lebt zur Hauptsache von der Landwirtschaft. Das ist ein hoher Prozentsatz, wenn man weiß, daß nur 7\% der finnischen Bodenfläche angebaut wird. Es ist erstaunlich, wie sich der Bauer in dem kargen Waldland dank unentwegter zäher Arbeit und Anspruchslosigkeit durchsetzen kann.

Naturgemäß konzentriert sich das Schwergewicht der Landwirtschaft auf die fruchtbaren südlichen Zonen, die ja auch am dichtesten bevölkert sind. Durch sorgfältige Sortenwahl und -zucht können jedoch bis weit nach Norden verschiedene Getreidearten angebaut werden.

Die Hauptzone des Weizens liegt in den fruchtbaren Tonebenen südlich des Salpausselkä und auf den Alandsinseln, wo noch viel Winterweizen angepflanzt wird. Die nördlichsten Sommerweizenfelder - allerdings nur noch vereinzelte - findet man hingegen im Landstrich Kemi-Tornio, also nahezu auf $66^{\circ}$ nördlicher Breite.

Der Hafer hat ein ähnliches Verbreitungsgebiet wie der Weizen, während der Roggen bis ins mittlere, die Gerste und Kartoffel sogar bis ins nördliche Lappland vordringen.

Eine absolute polare Grenze des Ackerbaus kann man nicht angeben. Auch im Eismeergebiet treiben Kolonisten und Lappen etwas Getreide- und Kartoffelbau, wenn auch mehr garten- als feldmäßig. Wirklich praktische Bedeutung wird dem Anbau der Feldfrüchte nur südlich der lappländischen Wasserscheide beigemessen. 
Im Süden, besonders in der Umgebung von Salo, wo die Zuckerfabrik steht, findet die Zuckerrübe zunehmende Verbreitung.

Trotzdem der Weizenbau in den letzten Jahren beträchtlich zugenommen hat, steht der Hafer immer noch an der Spitze mit einer Jahreserzeugung von 773 Millionen kg, gegenüber 262 Millionen kg Gerste, 235 Millionen kg Weizen und 132 Millionen $\mathrm{kg}$ Roggen.

Wegen der Verbilligung des ausländischen Getreides stellte sich um die Jahrhundertwende die Landwirtschaft auf die Viehhaltung um. Die bis zu jener Zeit allgemein übliche Dreifelderwirtschaft mit ihrem dreijährigen Turnus: Wintergetreide, Sommergetreide, Brache, mußte einer intensiveren Bebauungsmethode weichen. Man benötigte mehr Futter und stellte auf die Getreide-Futterbau-Wirtschaft mit Klee und Futterrüben um, während die Brache mancherorts ganz aufgegeben wurde.

\begin{tabular}{crrrrrr} 
Die Anbaufächen in 1000 ha & & & & $\begin{array}{c}\text { Ernte-Ertr.in } \\
\end{array}$ \\
\cline { 2 - 6 } Winterweizen & 1920 & 1930 & 1940 & & 1949 & $1000 \mathrm{t} 1949$ \\
Sommerweizen & 5,4 & 9,6 & 24,6 & 16,6 & 33,5 \\
Roggen & 3,3 & 4,4 & 116,7 & 179,6 & 289,1 \\
Gerste & 232,9 & 208,4 & 185,7 & 144,1 & 218,6 \\
Hafer & 116,0 & 115,4 & 113,8 & 122,5 & 181,1 \\
Gemischtkorn & 394,9 & 438,5 & 426,6 & 420,4 & 723,3 \\
Erbsen & 8,5 & 10,3 & 9,8 & 17,8 & 28,3 \\
Kartoffeln & 10,5 & 7,0 & 15,5 & 10,1 & 13,5 \\
Zuckerrüben & 71,3 & 71,3 & 80,7 & 85,6 & 115,7 \\
Total & 1,0 & 1,3 & 3,4 & 7,2 & 183,5 \\
& 843,8 & 866,2 & 976,8 & 1003,9 & 1786,6
\end{tabular}

Etwa die Hälfte der landwirtschaftlichen Einnahmen ist der Rinderhaltung zu verdanken. Die im Lande gezüchteten Rassen sind relativ klein, und mit einer durchschnittlichen jährlichen Milchleistung von $2000 \mathrm{~kg}$ pro Kuh, nicht besonders leistungsfähig. Dafür ertragen die Tiere die Unbilden der Witterung ohne Schaden und geben sich mit dem kargen Futter zufrieden. Die drei bekanntesten Rinderrassen Suomis sind die braune westfinnische, die weißbraune ostfinnische und die fast weiße nordfinnische Rasse.

Die Schaf- und Ziegenbestände sind seit den dreißiger Jahren zurückgegangen.

Das charakteristische Tier Lapplands, das Rentier, wird vorwiegend von den Lappen, seltener von Neusiedlern, gehalten. Es liefert Fleisch für den Eigenbedarf, wertvolles Fell und dient als Zugtier. Währenddem man 193996000 über ein Jahr alte Rentiere zählte, sollen es jetzt noch 80000 sein. Eine weitere Einnahmequelle sind schließlich die Pelztierfarmen, die meist von den Bauern als Nebenerwerb betrieben werden und der Silber- und Blaufuchszucht dienen.

\section{Viehbestand 1950}

\begin{tabular}{|c|c|c|c|c|c|}
\hline Rinder & & & & & \\
\hline Schafe . & . & . & & & \\
\hline Schweine & & & & & \\
\hline
\end{tabular}

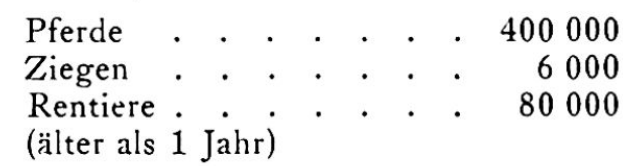

Bis weit hinauf gegen Norden hegen und pflegen die Bäuerinnen ihre meist kleinen Gärten. Die Finnen lieben Blumen, und so findet man allenthalben farbenfrohen Garten- und Zimmerschmuck. Überall gedeihen noch verschiedene Gemüsesorten, aber auch Johannis-, Stachel-, Erd- und Himbeeren werden gewöhnlich erfolgreich angepflanzt. Obstbäume sieht man nur im Süden, und zwar meistens in den Familiengärten bei den schmucken Häuschen. Einige klimaharte Apfelsorten reifen noch bis zum 65., Birnen, Pflaumen und Kirschen nur bis zum 62. Breitenkreis.

Seitdem Suomi selbständig ist, haben die landwirtschaftlichen Betriebsverhältnisse grundlegende Änderungen erfahren. Vorher waren die Besitzverhältnisse für die mei- 


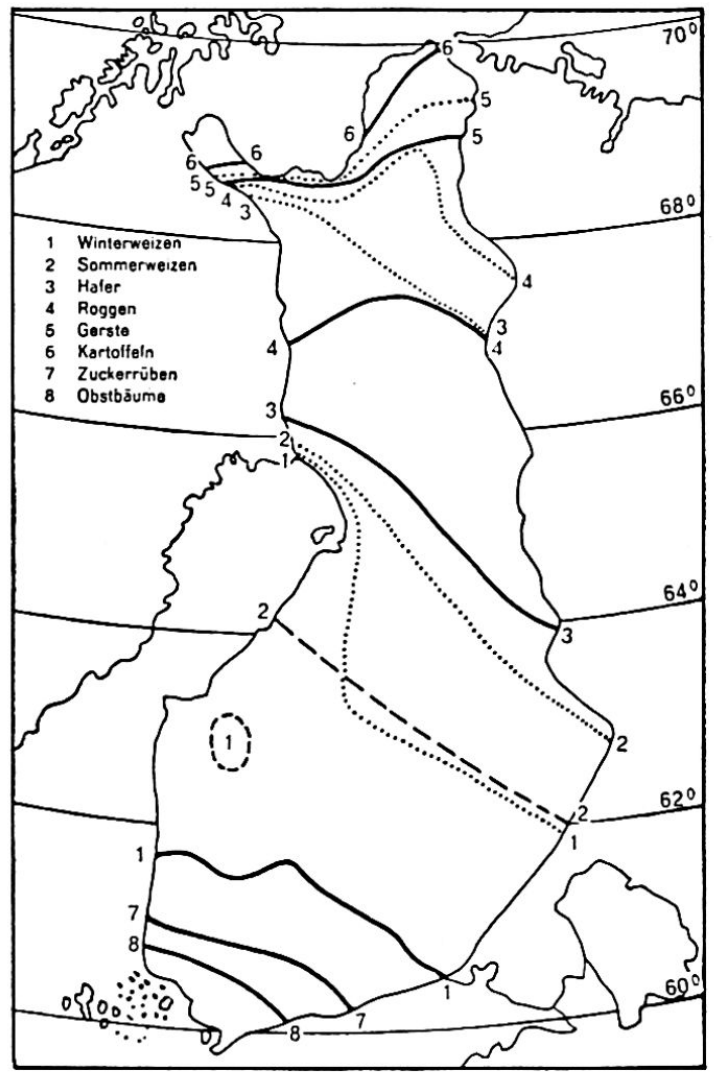

sten Bauern denkbar ungünstig. Nach den Statistiken von 1901 entfiel damals auf 110000 selbständige Ackerbauern, das waren $27 \%$ der ländlichen Bevölkerung, ein Areal von $173000 \mathrm{~km}^{2}$, worin der Waldbesitz eingerechnet war. Die restlichen $73 \%$ der Landbevölkerung besaßen keinen eigenen Boden! Sie waren arme Pächter oder Taglöhner und lebten oft in bitterer Not. Um bessere Verhältnisse zu erlangen, schuf das freie Finnland eine großzügige Sozialreform, die nach dem damaligen Präsidenten «Lex Kallio» genannt wird und sich das Ziel setzte, der besitzlosen Landbevölkerung zu eigenem Grund und Boden zu verhelfen. Staatliche Domänen und Großgrundbesitze, die gegen Entschädigung enteignet worden waren, teilte man in kleinere Besitzungen auf, und bis 1943 wurden 39000 Neugründungen durchgeführt. Zudem sind seither viele Bauern, die ihre Besitzungen in den an Rußland verlorenen Gebieten verlassen haben, auf ganz ähnliche Weise angesiedelt worden. So hat Suomi trotz harter Schicksalschläge in vorbildlicher Weise Innenkolonisation betrieben!

Landwirtschafts-Betriebe 1951

\begin{tabular}{rrr} 
Betriebsgröße in ha & Anzahl & in $\%$ \\
\hline-1 & 65000 & 18,4 \\
$1-5$ & 125000 & 35,3 \\
$5-10$ & 85000 & 24,0 \\
$10-25$ & 66000 & 18,7 \\
$25-50$ & 11000 & 3,1 \\
$50-100$ & 1500 & 0,4 \\
über 100 & 200 & 0,1 \\
\hline Total & 353700 & 100,0
\end{tabular}

Alle Anstrengungen der Landwirtschaft, die zum Teil nach amerikanischem Vorbild arbeitet, vermögen den Eigenbedarf des Landes nicht zu decken. Trotzdem wurden gewaltige Fortschritte erzielt, ist doch die Selbstyersorgung, die vor dem Ersten Weltkrieg $40 \%$ ausmachte, bis 1939 auf $87 \%$ angestiegen! 


\begin{tabular}{lcr} 
Produkt & $\begin{array}{r}1936-1939 \\
\text { (Jahresmittel) }\end{array}$ & 1954 \\
\hline Weizen & 210 & 235 \\
Roggen & 353 & 132 \\
Gerste & 189 & 262 \\
Hafer & 754 & 773 \\
Kartoffeln & 1337 & 1089 \\
Butter & 55 & 65 \\
Käse & 10 & 23 \\
Rindfleisch & 52 & 53 \\
Schweinefleisch & 56 & 67
\end{tabular}

Die Zeit der «schwendenden Bauern» ist schon längst vorbei. Der Wald wird nicht mehr rücksichtslos niedergebrannt und das Korn gleich darauf in die Asche gesät. Bis über den Polarkreis hinaus wird heute der Boden meist nach modernen Methoden bearbeitet, und kein anderes europäisches Land besitzt im Verhältnis zum Gesamtareal in diesen hohen nördlichen Breiten noch eine derart große Landwirtschaftsfläche wie das mutige Finnland!

\section{EINSAMES VOLK IM HINTERLAND BRASILIENS}

\section{RUdOLF STREIFF-BeCKER}

In Brasilien, der größten Republik Südamerikas, gibt es im Landesinneren noch ausgedehnte Landstriche, die unerforscht und nur teilweise und ungenau kartiert sind. Dort leben noch Sippen primitiver Indianer frei im zentralbrasilianischen Busch und Urwald, betreut vom staatlichen «Dienst zum Schutz der Indianer». Zwischen dem Wohngebiet der Indianer und den Küstenländern, wo die Kulturbevölkerung ihre intensive Tätigkeit entfaltet, liegt ein breiter, schwach bevölkerter Streifen Landes, mit wenig Ortschaften, deren Gründung zum Teil in die Kolonialzeit zurückreicht.

Im Jahre 1957 lief die Nachricht durch das Land, es sei im Hinterland (im «Sertão») des Staates Paraibo do Norte ein bisher unbekannter Indianerstamm entdeckt worden. Diese Kunde erregte nicht nur Ethnographen und Geographen, sondern auch weite Kreise der gebildeten Bevölkerung. Die bedeutende Zeitung «O Estado de São Paulo» beauftragte ihren Korrespondenten in der Stadt João Pessosa im Staat Paraiba do Norte, womöglich eine kompetente Persönlichkeit zur Auskundschaftung der fraglichen Gegend zu entsenden, was auch geschah. Die genannte Zeitung veröffentlichte in ihren Ausgaben vom 1., 3. und 4. September 1957 die Ergebnisse der Expedition, deren wesentliche Teile im folgenden wiedergegeben seien.

Die Nachricht von der Existenz eines unbekannten, namenlosen Völkleins war nach der Stadt Santa Luzia durch einen Mann, namens Julio VieIRA gebracht worden. Dieser, ein pensionierter Unteroffizier der brasilianischen Armee, war einst aus Abenteuerlust in den Sertão gezogen. Er war bei ungefähr 6 bis $7 \mathrm{Grad}$ südl. Breite, in der Serra do Talhado, auf einsam lebende Menschen dunkler Hautfarbe gestoßen und als Lehrer und Berater unter ihnen geblieben. Später kehrte er jedoch entmutigt wieder nach Santa Luzia zurück und bewirkte durch seine Erzählungen die eingangs erwähnte Nachricht von der Entdeckung unbekannter Indianer. In Santa Luzia kennt man nun schon seit langem gewisse Caboclos, die gelegentlich aus dem Inneren zu den Jahrmärkten der Stadt kommen, durch ihre eigenartige Kleidung auffallen, stets ein Buschmesser mit langem Griff im Gürtel tragen, dem Zuckerrohrschnaps zugetan sind und unter dessen Wirkung sehr rauflustig werden. Sie bringen auf den Jahrmarkt eigenartige Tongefäße, die sie gegen fremde Waren eintauschen. Ihre blau- 